\title{
Nerve growth factor upregulates sirtuin 1 expression in cholestasis: a potential therapeutic target
}

\author{
Ming-Shian Tsai ${ }^{1,2}$, Po-Huang Lee ${ }^{1}$, Cheuk-Kwan Sun ${ }^{3}$, Ting-Chia Chiu ${ }^{1}$, Yu-Chun Lin ${ }^{1}$, I-Wei Chang ${ }^{4}$, \\ Po-Han Chen ${ }^{3}$ and Ying-Hsien $\mathrm{Kao}^{3}$
}

This study investigated the regulatory role of nerve growth factor (NGF) in sirtuin 1 (SIRT1) expression in cholestatic livers. We evaluated the expression of NGF and its cognate receptors in human livers with hepatolithiasis and the effects of NGF therapy on liver injury and hepatic SIRT1 expression in a bile duct ligation (BDL) mouse model. Histopathological and molecular analyses showed that the hepatocytes of human diseased livers expressed NGF, proNGF (a precursor of NGF), TrkA and p75NTR, whereas only p75NTR was upregulated in hepatolithiasis, compared with non-hepatolithiasis livers. In the BDL model without NGF therapy, p75NTR, but not TrkA antagonism, significantly deteriorated BDL-induced liver injury. By contrast, the hepatoprotective effect of NGF was abrogated only by TrkA and not by p75NTR antagonism in animals receiving NGF therapy. Intriguingly, a positive correlation between hepatic SIRT1 and NGF expression was found in human livers. In vitro studies demonstrated that NGF upregulated SIRT1 expression in mouse livers and human Huh-7 and rodent hepatocytes. Both NGF and proNGF induced protective effects against hydrogen peroxide-induced cytotoxicity in Huh-7 cells, whereas inhibition of TrkA and p75NTR activity prevented oxidative cell death. Mechanistically, NGF, but not proNGF, upregulated SIRT1 expression in human Huh-7 and rodent hepatocytes via nuclear factor (NF)-кB activity, whereas NGF-induced phosphoinositide-3 kinase/Akt, extracellular signal-regulated kinase and NF-KB signaling and SIRT1 activity were involved in its hepatoprotective effects against oxidative injury. These findings suggest that pharmacological manipulation of the NGF/SIRT1 axis might serve as a novel approach for the treatment of cholestatic disease.

Experimental \& Molecular Medicine (2018) 50, e426; doi:10.1038/emm.2017.235; published online 12 January 2018

\section{INTRODUCTION}

Hepatolithiasis, defined as the presence of gallstones within the intrahepatic biliary ducts, is more prevalent in the Asia-Pacific region than in Western countries. ${ }^{1-3}$ The prevalence of hepatolithiasis ranges from $30 \%$ to $50 \%$ among patients with cholelithiasis in Eastern regions ${ }^{4,5}$ and from $0.6 \%$ to $1.3 \%$ in Western regions. ${ }^{4}$ However, increased immigration from endemic areas has resulted in an elevated incidence of hepatolithiasis in the West. ${ }^{4,6}$ The clinical manifestations of hepatolithiasis include repeated attacks of acute bacterial cholangitis, the subsequent formation of further stones and strictures in the biliary system and persistent obstructive jaundice. ${ }^{7}$ If left untreated, hepatolithiasis can lead to irreversible liver failure and mortality. ${ }^{7}$ So far, there is still a lack of an effective medical treatment to alleviate cholestatic liver injury in hepatolithiasis except for surgery.
We previously demonstrated that nerve growth factor (NGF) was upregulated in cholestatic liver models and could protect hepatocytes against oxidative stress, ${ }^{8}$ which is crucial for cholestatic liver injury. ${ }^{9}$ Therefore, the identification and therapeutic targeting of NGF signaling should be considered as an alternative strategy to treat hepatolithiasis. To discover new treatment options for hepatolithiasis, we first investigated the expression of NGF, proNGF (the precursor of NGF), and their receptors, TrkA and p75NTR, in human liver samples. The expression levels of these proteins were correlated with several clinical parameters. In addition, we performed in vitro mechanistic studies to explore the possible molecular mechanisms underlying the antioxidative effects of NGF and proNGF, including nuclear factor (NF)- $\kappa B$ and sirtuin 1 (SIRT1). Finally, we tested the therapeutic effects of NGF in vivo and studied whether TrkA or p75NTR is the

\footnotetext{
${ }^{1}$ Department of Surgery, E-Da Hospital, I-Shou University, Kaohsiung, Taiwan; ${ }^{2}$ The School of Medicine for Post-Baccalaureates, I-Shou University, Kaohsiung, Taiwan; ${ }^{3}$ Department of Medical Research, E-Da Hospital, I-Shou University, Kaohsiung, Taiwan and ${ }^{4}$ Department of Pathology, E-Da Hospital, I-Shou University, Kaohsiung, Taiwan

Correspondence: Dr M-S Tsai, Department of Surgery, E-Da Hospital, I-Shou University, Kaohsiung 82445, Taiwan.

E-mail: dtsurgq6@gmail.com

or Dr Y-H Kao, Department of Medical Research, E-Da Hospital, I-Shou University, Kaohsiung 82445, Taiwan.

E-mail: danyhkao@gmail.com

Received 12 December 2016; revised 13 July 2017; accepted 16 July 2017
} 
receptor essential for the effects of NGF in cholestatic liver injury.

\section{MATERIALS AND METHODS}

\section{Reagents}

TrkA blocker, GW441756 (G3420), and kinase inhibitors, including LY294002 (L9908), PD98059 (P-215), pyrrolidine dithiocarbonate (PDTC, P8765), SIRT1 agonist resveratrol (R5010) and antagonist EX-527 (E7034), were purchased from Sigma-Aldrich (St Louis, MO, USA). A p75NTR blocker, PD90780, was purchased from Axon Medchem (Groningen, The Netherlands). Recombinant NGF and proNGF peptides were purchased from AbD Serotec (PMP04Z; Oxford, UK) and Alonmone Labs (N-250; Jerusalem, Israel), respectively.

\section{Human samples}

The study included four patients with hepatocellular carcinoma and five patients with hepatolithiasis (four with the liver affected on the left side, one on the right side) undergoing liver resection with surgical procedures similar to those previously described. ${ }^{10}$ All of the human liver tissues were collected in accordance with the Declaration of Helsinki of 1975, as revised in 1983, and the procedures were approved by the Institutional Review Board of E-Da Hospital (Approval No. EMRP14103N) with written informed consent from all of the subjects. The liver samples (non-tumor part) from patients with hepatic tumors were termed the non-hepatolithiasis controls (NHCs). For hepatolithiasis patients, liver tissues with (lithiasis side) and those without (contralateral side) calculi were collected by wedge liver biopsy and were subjected to further study. Preoperative serum biochemical parameters, including aspartate aminotransferase (AST), alanine aminotransferase (ALT), direct bilirubin (DB) and total bilirubin (TB), were retrospectively obtained.

\section{Histopathology and immunohistochemistry (IHC) staining Formalin-fixed and paraffin-embedded human and mouse livers were sectioned and used for hematoxylin and eosin and IHC staining, as previously described. ${ }^{8}$ Deparaffinized and rehydrated sections were treated for antigen retrieval and incubated with primary antibodies at $4{ }^{\circ} \mathrm{C}$ overnight. Rabbit monoclonal antibodies purchased from Abcam (clone E51, Cambridge, MA, USA) were used to localize NGF (ab52918), TrkA (ab76291), p75NTR (ab52987) and the cleaved peptides of poly(ADP-ribose) polymerase (PARP). Anti-proNGF was purchased from Alomone Labs (Jerusalem, Israel) (ANT-005). The antigen location in tissue sections was visualized with an horseradish peroxidase-linked polymer Envision detection system (DAKO, Glostrup, Denmark), followed by counterstaining with hematoxylin. Normal liver sections were treated with equimolar concentrations of isotype-matched normal $\operatorname{IgG}$ as negative controls.}

\section{Western blottings}

Protein extraction, sodium dodecyl sulfate-polyacrylamide gel electrophoresis and immunodetection were performed as previously described. ${ }^{8}$ For immunodetection, antibodies against SIRT1 and the pro-peptide and active forms of caspase- 3 and PARP, as well as the total and phosphorylated forms of Akt (Ser473; no. 4685), extracellular signal-regulated kinase 1/2 (ERK1/2) (Thr202/Tyr204; no. 9101) and NF-KB p65 (Ser536; no. 3033), were purchased from Cell Signaling (Danvers, MA, USA). Antibodies against TrkA (ab76291) and p75NTR (ab52987) were acquired from Abcam. Anti-proNGF was purchased from Alomone Labs (ANT-005). Anti-NGF
(AB1528SP) and $\beta$-Actin (MAB1501) was acquired from Millipore (Temecula, CA, USA). For semiquantitative analyses, relative protein levels were expressed as fold induction compared with the negative control by normalizing the density of the protein of interest to an internal control.

\section{Reverse transcription and quantitative PCR (RT-qPCR)}

Total RNA was extracted using TRIzol reagent (Invitrogen, Carlsbad, CA, USA) and was subjected to RT-qPCR detection using genespecific primers (Supplementary Table S1) and a previously described protocol. $^{8}$

\section{Animals and treatment}

Eight-week-old ICR male mice were raised with ad libitum access to food at $20-22{ }^{\circ} \mathrm{C}$ with a 12 -h light-dark cycle in the Animal Center of I-Shou University. All of the animal handling procedures were approved by the Institute of Animal Care and Use Committee at E-Da Hospital (Approval Nos. IACUC-102033 and -103030) and were performed in accordance with the Guide for the Care and Use of Laboratory Animals (NIH Publication No. 85-23, revised 1996). Mice were randomly assigned to experimental groups. Cholestatic liver injury was surgically induced by common bile duct ligation (BDL), as previously described. ${ }^{8,11}$ For NGF receptor (NGFR) antagonist treatment, GW441756 dissolved in phosphate-buffered saline (PBS) and PD90780 dissolved in dimethyl sulfoxide (DMSO) were intraperitoneally administered at $1 \mathrm{mg} \mathrm{kg}^{-1} \mathrm{day}^{-1}$ immediately after BDL surgery. Equivalent volumes of PBS and DMSO were used for respective solvent controls. For NGF therapy and NGF neutralization treatment, recombinant NGF at $1 \mathrm{mg} \mathrm{kg}^{-1}$ and rabbit anti-NGF neutralizing antibody (AB1526SP, Millipore) at $2 \mathrm{mg} \mathrm{kg}^{-1}$ were intraperitoneally injected twice weekly. Equivalent volumes and doses of PBS and species- and isotype-matched normal IgG (N-IgG) were used as vehicle controls. Fourteen days after BDL surgery, serum was collected for biochemical measurement of AST, ALT and TB by a clinical automatic analyzer (Department of Laboratory Medicine, E-Da Hospital, Kaohsiung, Taiwan). Liver tissues were dissected and aliquoted for protein and histological analyses.

\section{Apoptotic detection by TUNEL staining}

Formalin-fixed and paraffin-embedded mouse liver sections were subjected to a TUNEL (terminal deoxinucleotidyl transferasemediated dUTP-fluorescein nick end labeling)-based in situ cell death detection assay (Roche, Mannheim, Germany), according to the manufacturer's protocol. The sections were counterstained with hematoxylin. The nuclear TUNEL signals were quantified by counting at least five randomly selected images per liver section under highpower light field microscopy. The results were shown as the percentage of TUNEL-positive cells, compared with the total parenchymal cells.

\section{Cell culture and viability assay}

For cytokine stimulation experiments, primary hepatocytes were isolated from adult male Sprague-Dawley rats and ICR mice using a two-step collagenase perfusion method, as previously described. ${ }^{12}$ Primary hepatocytes were seeded onto plates precoated with type I collagen at a density of $5 \times 10^{5}$ cells per well. For the mechanistic study, a hepatocyte cell line, clone-9, was purchased from the Bioresource Collection and Research Center (BCRC no. 60201, Hsin-Chu, Taiwan) and maintained in F-12K medium (Sigma) supplemented with $10 \%$ heat-inactivated fetal calf serum (Invitrogen, 
Logan, UT, USA) and standard antibiotics. ${ }^{11}$ For determination of cell viability, MTT (3-[4,5-dimethylthiazol-2-yl]-2,5 diphenyl tetrazolium bromide)-based cellular assay was performed as previously described. ${ }^{13}$

\section{Enzyme-linked immunosorbent assay}

Serum tumor necrosis factor- $\alpha$ and interleukin- 6 levels were measured using commercially available enzyme-linked immunosorbent assay (ELISA) kits, according to the manufacturer's instructions (Biolegend, San Diego, CA, USA).

\section{Statistical analysis}

In vivo and in vitro results are presented as the mean \pm s.e.m. and mean \pm s.d., respectively. Significant differences between groups were determined by one-way analysis of variance followed by Bonferroni's post hoc test. Spearman's correlation analysis was performed using the PASW Statistics software (Windows version 18.0, SPSS Inc., Chicago, IL, USA). A $P$-value $<0.05$ was considered statistically significant.

\section{RESULTS}

Expression of NGF, proNGF and their receptors in human livers with hepatolithiasis

To determine changes in expressional of NGF, proNGF and their cognate receptors (TrkA and p75NTR) in human cholestatic livers, five patients with hepatolithiasis were enrolled for histological and molecular studies. Four liver tissue samples from patients without hepatolithiasis were regarded as NHCs. The clinicopathological characteristics of all of the patients and semiquantitative measurements of liver proteins are shown in Supplementary Table S2. Liver protein extracts were subjected to western blotting detection of NGF, proNGF and their receptors (Figure 1a). Densitometry indicated no significant elevation of NGF in the livers with hepatolithiasis and lower proNGF abundance in the contralateral regions of diseased livers (Figure 1b). In addition, the protein expression of p75NTR, but not TrkA, significantly increased in contralateral hepatolithiasis samples compared with that in the NHC livers (Figure 1c), although RT-qPCR analysis revealed no prominent changes in the ligand and receptor mRNA levels (Figure 1d). Despite the limited number of human samples in this study, Spearman's correlation analysis suggested that serum DB levels had an inverse relationship with hepatic NGF (Figure 1e) but not with proNGF expression (Figure 1f). Moreover, hepatic expression of both NGFRs displayed positive correlations with serum DB levels (Supplementary Figures S1A and B), whereas hepatic NGF expression decreased with age (Supplementary Figure $\mathrm{S1C}$ ). These findings indicated a protective role for NGF in hepatolithiasis.

To scrutinize the histological localization of NGF, proNGF and both NGFRs in cholestasis-injured human livers, we next examined their histological distribution using IHC staining. The results clearly showed that NGF, proNGF and both NGFRs, TrkA and p75NTR, were mainly located in the parenchymal hepatocytes and biliary ductular epithelial cells of both control and diseased livers (Figure 2). Intense signals of both NGF and proNGF were notably seen in the parenchymal areas of lithiasis livers with prominent fatty changes, while dispersed TrkA and p75NTR signals were detected not only in the hepatocytes of both control and diseased livers but also in the infiltrated cells in the contralateral regions of lithiasis tissues. The parenchymal localization of both NGFRs was similar to the distribution patterns observed in the murine livers with BDL-induced cholestatic injury (Supplementary Figure S2). However, no discernible difference in spatial localization was found between NHC and hepatolithiasis liver tissues in both regions.

\section{Significance of TrkA and p75NTR in BDL-induced liver injury without NGF therapy}

To explore the roles of both NGFRs in the pathogenesis of cholestatic liver disease, ICR mice were treated with antagonists for the NGFRs (GW441756 for TrkA and PD90780 for p75NTR) in conjunction with BDL-induced liver injury. Biochemical analyses indicated that the administration of a p75NTR antagonist, but not a TrkA antagonist, significantly deteriorated the serum levels of AST (Figure 3a) and ALT (Figure $3 \mathrm{~b}$ ) in BDL-treated mice compared with their respective solvent controls, despite no change in TB levels (Figure 3c). NGF treatment remarkably attenuated TB elevations in mice with cholestatic liver injury. However, ELISA data showed that both antagonists ameliorated elevations in tumor necrosis factor- $\alpha$ (Figure 3d) and interleukin-6 (Figure 3e) in mouse serum. To confirm the severity of liver injury, histological hematoxylin and eosin staining (Figure 3f) followed by morphometric quantification (Figure $3 \mathrm{~g}$ ) corroborated that only antagonism of p75NTR, but not of TrkA, significantly regulated the severity of cholestasis-induced liver necrosis.

To examine whether pro-apoptotic mediators were involved in cholestatic liver injury, the total and cleaved peptides of caspase- 3 and PARP in mouse liver extracts were detected by western blotting (Figure 4a). The density analysis of cleaved caspase- 3 peptides showed that, although remarkable elevation of caspase- 3 cleavage was noted in the TrkA antagonism group (Figure 4b), p75NTR antagonism after BDL surgery significantly increased the active PARP level in cholestatic livers, compared with the DMSO solvent controls (Figure 4c). Consistently, IHC staining using antibodies against the active form of PARP confirmed that p75NTR blockade significantly increased the presence of active PARP (Figures $4 \mathrm{~d}$ and e). Furthermore, antagonism of p75NTR, but not of TrkA, remarkably increased apoptotic events in BDL-treated mice as revealed by TUNEL staining (Figure 4f) and subsequent quantitative analysis (Figure $4 \mathrm{~g}$ ).

\section{Significance of TrkA and p75NTR in BDL-induced liver injury with NGF therapy}

To determine which receptor was responsible for the NGF-mediated hepatoprotective effect in cholestatic injury, the mice receiving BDL surgery were simultaneously administered recombinant NGF peptides and daily injection of either NGFR antagonist. Biochemical analyses showed no alterations in the serum levels of AST (Figure 5a), ALT (data not shown) 
a

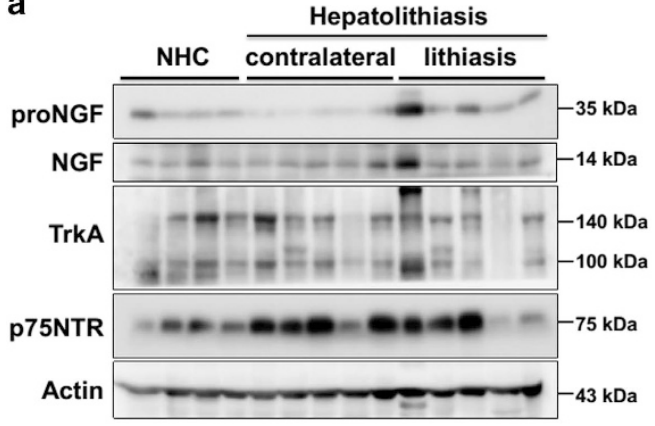

b

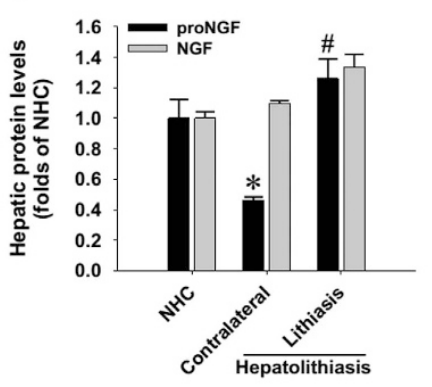

C

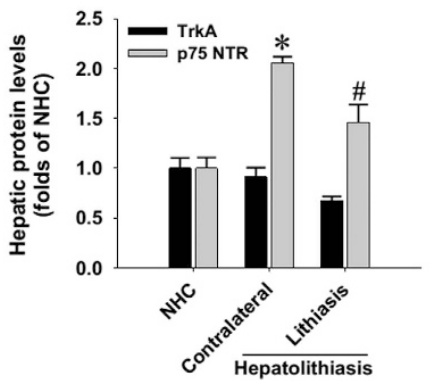

d

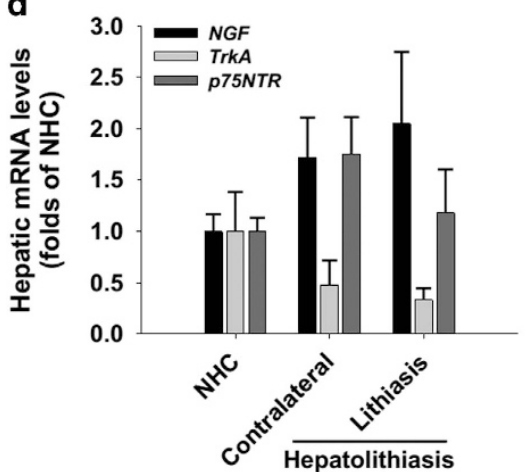

e

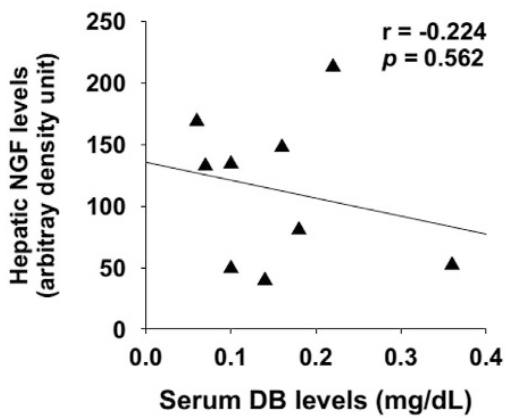

f

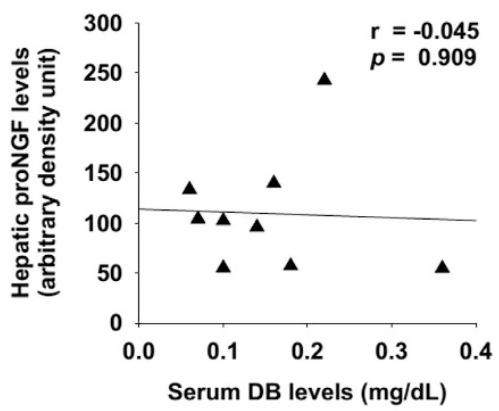

Figure 1 Expression of NGF, proNGF and their cognate receptors, TrkA and p75NTR, in human livers with hepatolithiasis. (a) Hepatolithiasis samples $(n=5)$ included lithiasis and contralateral liver tissues. Paralesional protein extracts from patients with hepatocellular carcinoma were the non-hepatolithiasis controls (NHC, $n=4)$ and were subjected to western blotting detection of NGF, proNGF, TrkA and p75NTR levels. The scan density data of NHC and hepatolithiasis groups are shown in Supplementary Tables S3 and S4, respectively. Densitometrical analyses of the expression levels of NGF and proNGF (b), as well as those of TrkA and p75NTR (c), are shown as the mean \pm s.e.m. of their ratios to internal actin controls. ${ }^{*} P<0.05$ vs NHC levels, ${ }^{\#} P<0.05$ vs contralateral levels. (d) RT-qPCR detection of NGF, TrkA and p75NTR mRNA levels in human livers with hepatolithiasis. Spearman's correlation analysis of the relationships of hepatic NGF (e) and proNGF expression (f) with serum total bilirubin levels.

and TB (Figure 5b) in BDL-treated mice with administration of both antagonists, compared with respective solvent controls. However, ELISA data showed that TrkA antagonism significantly aggravated, whereas p75NTR antagonism ambiguously alleviated, the elevation of serum interleukin-6 (Figure 5c) but not of tumor necrosis factor- $\alpha$ (data not shown) in BDL-treated mice. Histological staining (Figure 5d) and morphometric quantification (Figure 5e) indicated that only antagonism of TrkA, but not of p75NTR, significantly increased the necrotic area in the cholestatic livers. Next, western blotting detection of pro-apoptotic mediators (Figure 6a) and subsequent densitometrical analysis clearly showed that TrkA antagonism significantly increased the cleavage of caspase-3 (Figure 6b) and PARP peptides (Figure 6c) in cholestatic livers, whereas p75NTR antagonism decreased the peptide cleavage. IHC staining of active PARP showed that TrkA inhibition significantly increased PARP activation, but no difference was noted between p75NTR antagonism and its solvent control groups (Figures 6d and e). Supportive of the IHC data, TUNEL staining results demonstrated that the activity of TrkA, but not of p75NTR, was significantly involved in apoptogenesis in the cholestatic mice receiving NGF therapy (Figures $6 f$ and g).

\section{Expression of SIRT1 in human hepatolithiasis and mouse} cholestatic livers

Given that SIRT1 has been shown to protect neuronal cells against oxidative stress, ${ }^{14}$ we next examined the possibility that NGF exerts its hepatoprotective effects via SIRT1 in both clinical and experimental settings. Although western blotting revealed no significant change in hepatic SIRT1 expression between human NHC and hepatolithiasis liver tissues (Figure 7a), RT-qPCR analysis indicated that SIRT1 mRNA levels remarkably decreased in the contralateral regions of hepatolithiasis livers (Figure 7b). Spearman's correlation analysis intriguingly showed that the hepatic SIRT1 expression was positively and strongly correlated with NGF levels but moderately with proNGF levels (Figure 7c). In addition, the hepatic SIRT1 levels decreased with age (Supplementary Figure S1D) and showed a significantly positive correlation with p75NTR, but not TrkA, expression (Supplementary Figures S1E and F). Moreover, IHC staining results showed both nuclear and cytoplasmic localization of SIRT1 in the parenchymal hepatocytes of diseased livers (Figure $7 d$ ). To examine the positive role of NGF in the regulation of hepatic SIRT1 expression in animals, the liver extracts of the mice receiving BDL surgery and simultaneous treatment with 


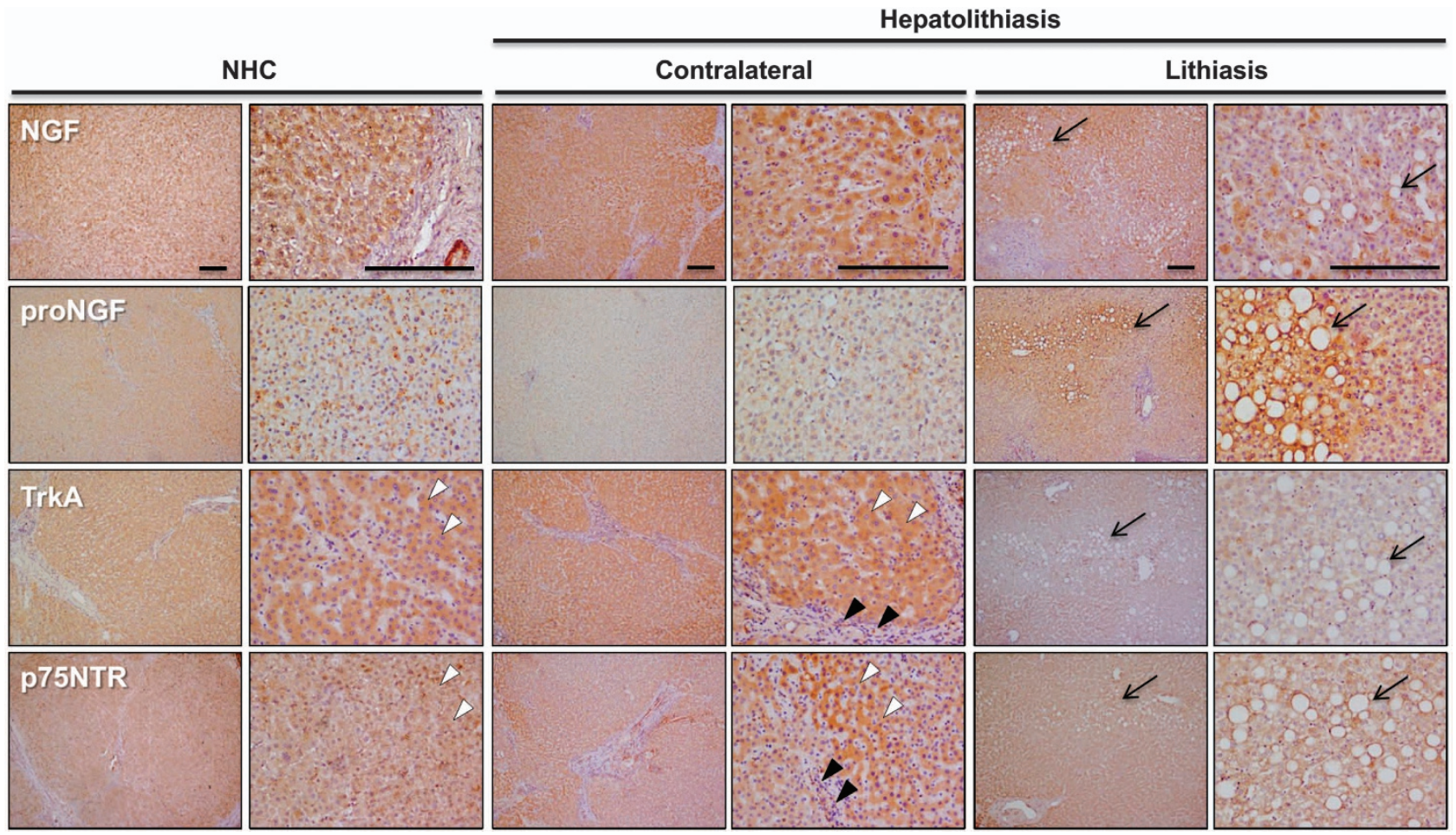

Figure 2 Histological localization of NGF, proNGF and their cognate receptors, TrkA and p75NTR, in parenchymal hepatocytes of human livers with hepatolithiasis. Liver sections of lithiasis and contralateral parts in hepatolithiasis liver tissues $(n=5)$ and paralesional sections of hepatocellular carcinoma livers $(n=4)$ as non-hepatolithiasis control (NHC) underwent formalin-fixation, tissue-processing and paraffinembedding procedures. Tissue sections were subjected to immunohistochemical staining for NGF, proNGF, TrkA and p75NTR. Scale bars $=100 \mu \mathrm{m}$. Arrows indicate characteristic fatty changes in lithiasis tissues, while white and black arrowheads indicate immunoreactive signals in the hepatocytes and the infiltrated cells, respectively.

recombinant NGF peptides, neutralizing antibodies or respective solvent controls were subjected to western blotting analysis. The results showed that NGF supplementation remarkably increased hepatic SIRT1 levels in the injured livers, whereas administration of NGF-neutralizing antibodies conversely reduced SIRT1 expression, compared with corresponding PBS and IgG controls (Figure 7e). Similar to the distribution pattern in human livers, NGF therapy increased both nuclear and cytoplasmic SIRT1 expression in BDL-injured mouse livers (Figure 7f). These findings strongly suggested a positive role of NGF in the regulation of hepatic SIRT1 expression.

\section{NGF-induced signaling and SIRT1 upregulation protect hepatocytes against oxidative injury}

To further clarify the regulatory role of NGF in SIRT1 expression, the protein lysates of human Huh-7 hepatocytes with NGF or proNGF stimulation were analyzed by western blotting. The results demonstrated that NGF, but not proNGF, significantly upregulated SIRT1 protein expression in Huh-7 cells (Figure 8a). The stimulatory effect of NGF on SIRT1 expression was confirmed in cultured primary rat and mouse hepatocytes (Figure 8b). To delineate the NGF-stimulated signaling profiles, western blotting analysis confirmed that exogenous NGF triggered Akt, ERK1/2 and NF- $\mathrm{BB}$ hyperphosphorylation in human Huh-7 hepatocytes (Figure 8c), consistent with the kinetic signaling profiles noted in clone-9 hepatocytes (Supplementary Figures S4A and B). To determine the involvement of NF- $\mathrm{KB}$ activity in NGF-upregulated SIRT1 expression, PDTC pretreatment followed by NGF stimulation significantly abrogated NGF-induced SIRT1 upregulation (Figure 8d), supporting the regulatory role of NF- $\mathrm{KB}$ in hepatic SIRT1 expression.

To determine the receptor responsible for NGF-elicited signaling activation, clone-9 rat hepatocytes were pretreated with respective NGFR antagonists, followed by NGF stimulation. NGF-evoked Akt, ERK1/2 and NF-кB hyperphosphorylation under oxidative stress-free conditions was mediated by TrkA, but not p75NTR, activity (Supplementary Figure S3). Receptor-blockade experiments indicated that TrkA mediated ERK activation, whereas p75NTR initiated NF- $\mathrm{KB}$ cascade (Supplementary Figures S4C and D). The blockade of both NGFRs enhanced $\mathrm{H}_{2} \mathrm{O}_{2}$-induced PARP cleavage (Supplementary Figure S4E), cytotoxicity (Supplementary Figure S4F) and morphological deterioration (Supplementary Figure S5) in clone-9 cells. These results suggested that both NGFRs substantially participated in NGF-induced hepatoprotection in vitro.

To verify the contribution of NGF-induced signaling and SIRT1 activation to its hepatoprotective effect in vitro, western blotting analysis showed that treatment with 

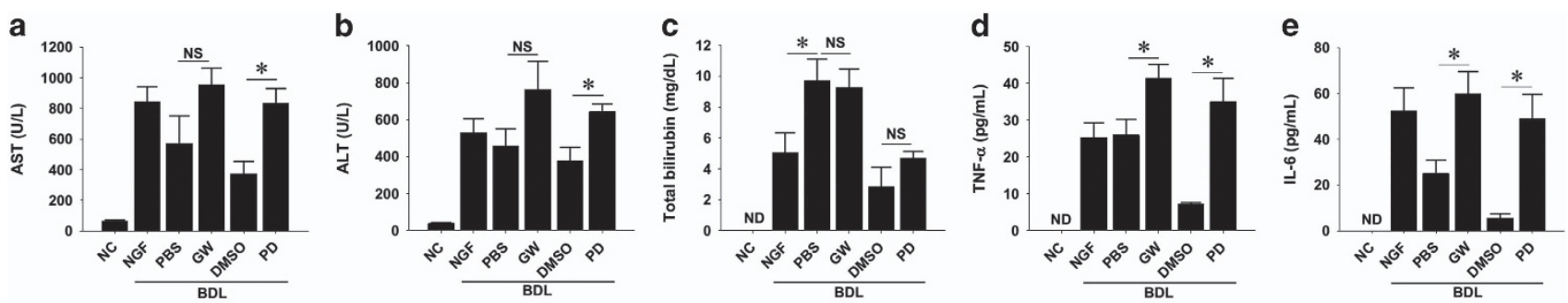

f
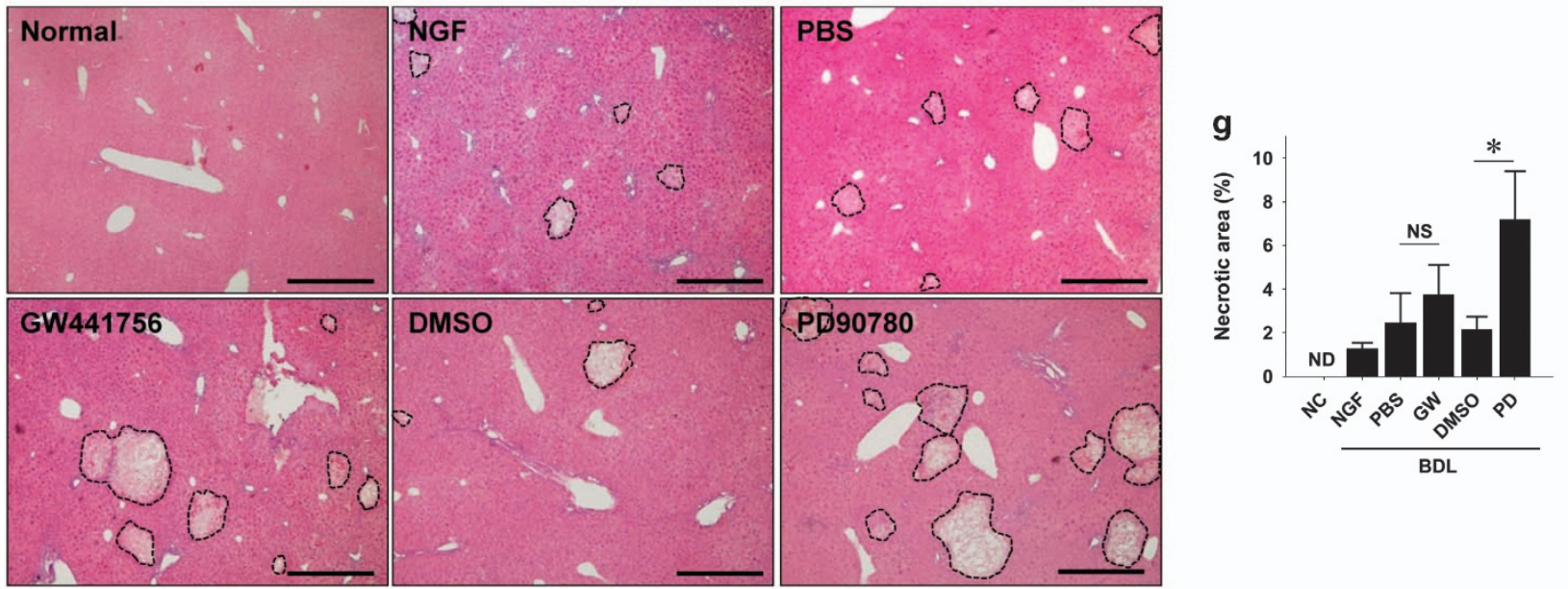

Figure 3 Effects of TrkA and p75 NTR blocker treatments on bile duct ligation (BDL)-induced mouse liver injury. The ICR mice receiving $\mathrm{BDL}$ surgery were intraperitoneally administered either recombinant NGF $(n=6)$ twice weekly or PBS $(n=6)$, GW441756 (GW; $n=6)$, DMSO $(n=3)$ or PD90780 (PD; $n=6$ ) daily. At 14 days postoperation, serum levels of AST (a), ALT (b) and total bilirubin (c) were biochemically measured, whereas tumor necrosis factor (TNF)- $\alpha$ (d) and interleukin (IL)-6 (e) levels in serum were analyzed by ELISA. The $\mathrm{H} \& \mathrm{E}$ staining results of mice with BDL-induced cholestatic injury or simultaneous treatments show the hepatic necrotic regions in dashed circles (f), quantified by morphometric analysis (g). Scale bar $=1000 \mu \mathrm{m}$. All of the data are shown as the mean \pm s.e.m. ND, not detectable. NS, not significant. ${ }^{*} P<0.05$ between the indicated groups.

resveratrol, a well-known SIRT1 agonist, dramatically ameliorated $\mathrm{H}_{2} \mathrm{O}_{2}$-increased caspase- 3 and PARP cleavage, whereas treatment with EX-527, a SIRT1 antagonist, conversely potentiated the activation of pro-apoptotic caspase- 3 and PARP in human Huh-7 hepatocytes (Figure 8e, Supplementary Figure S6). Cellular viability assays consistently demonstrated that resveratrol significantly enhanced the viability of $\mathrm{H}_{2} \mathrm{O}_{2}$ exposed Huh-7 cells, whereas the depletion of SIRT1 activity by EX-527 completely abrogated the NGF-elicited hepatoprotective effect (Figure 8f). Moreover, kinase inhibition treatments showed that NGF-evoked Akt, ERK1/2 and NF- $\mathrm{KB}$ signaling activities were all involved in its hepatoprotective effects on Huh-7 (Figures 8e and f) and clone-9 hepatocytes (Supplementary Figure S4G). SIRT1-dependent NGF-mediated hepatoprotection against oxidative injury was confirmed in cultured rat primary hepatocytes (Supplementary Figure S7). In addition, proNGF similarly evoked signaling activation of Akt, ERK1/2 and NF- $\mathrm{KB}$ in Huh-7 cells, also contributing to its hepatoprotective effects (Supplementary Figure S8). These findings strongly supported the theory that SIRT1 is an important effector molecule lying downstream of NGF signaling cascades in hepatocytes under oxidative stress.

\section{DISCUSSION}

The present study is the first to investigate the significance of NGF signaling in the pathophysiological mechanisms of hepatolithiasis. Moreover, we investigated the idea that the manipulation of NGF signaling could be a therapeutic target for hepatolithiasis-induced liver injury. We found that p75NTR, but not NGF, showed increased expression in liver samples obtained from patients with hepatolithiasis. Interestingly, not only $\mathrm{NGF}^{8}$ but also proNGF ${ }^{15}$ protected hepatocytes from oxidative injury in vitro, whereas blockade of either TrkA or p75NTR ameliorated the hepatoprotective effects of NGF and proNGF in vitro. Further in vivo experiments suggested that exogenous NGF administration was able to inhibit hepatocyte apoptosis in cholestatic liver injury. To further determine the significance of TrkA and p75NTR signaling in cholestatic liver injury, we pharmacologically blocked the two receptors in vivo with and without exogenous NGF administration. Without exogenous NGF, blockage of p75NTR worsened cholestatic liver injury, whereas an antagonist of TrkA abrogated the hepatoprotective effect when exogenous NGF was administered. Moreover, in vitro findings demonstrated a significant role of NGF signaling in mediating hepatic SIRT1 expression in hepatocytes, and the induced signal and 
SIRT1 activities were involved in its hepatoprotective effects. Therefore, the NGF/SIRT1 axis might be a potential therapeutic target for treating liver injury caused by hepatolithiasis.
Understanding the causes of liver damage in cholestatic disease is critical for developing treatment strategies to improve prognosis. Persistent cholangitis and the presence of gallstones a

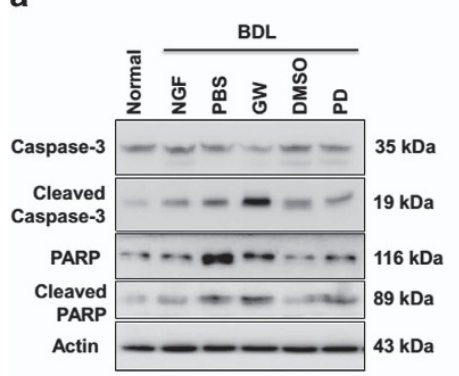

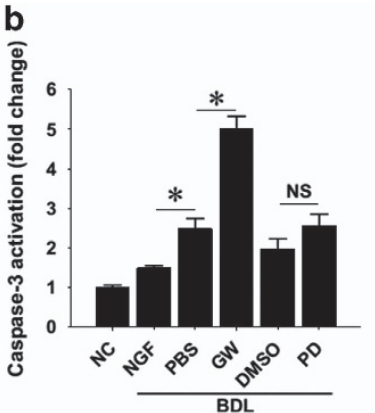

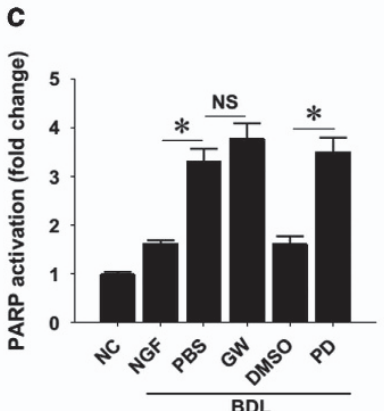

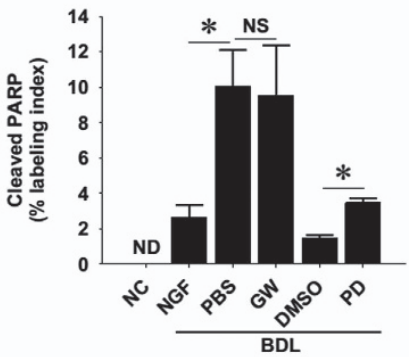

d
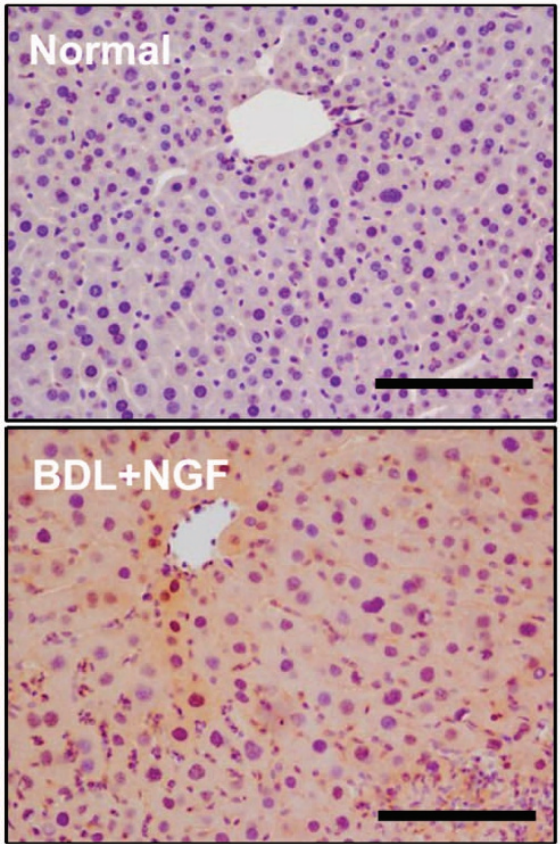

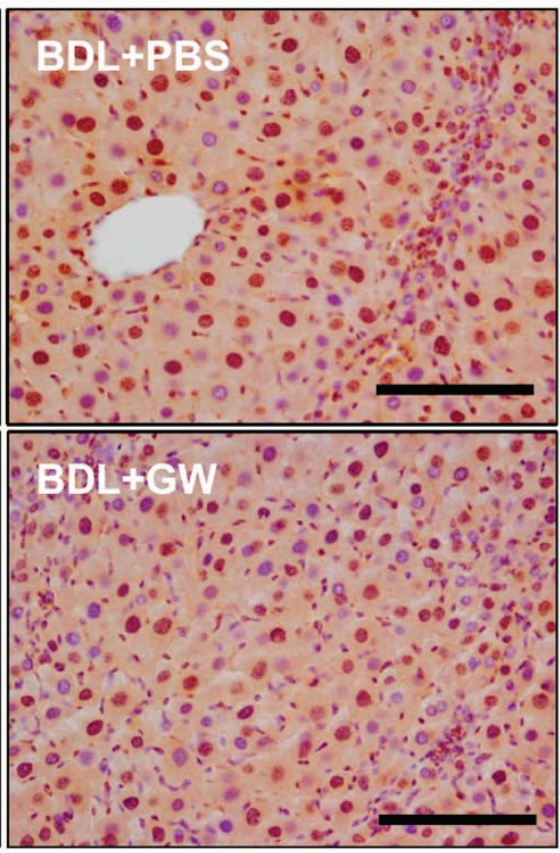

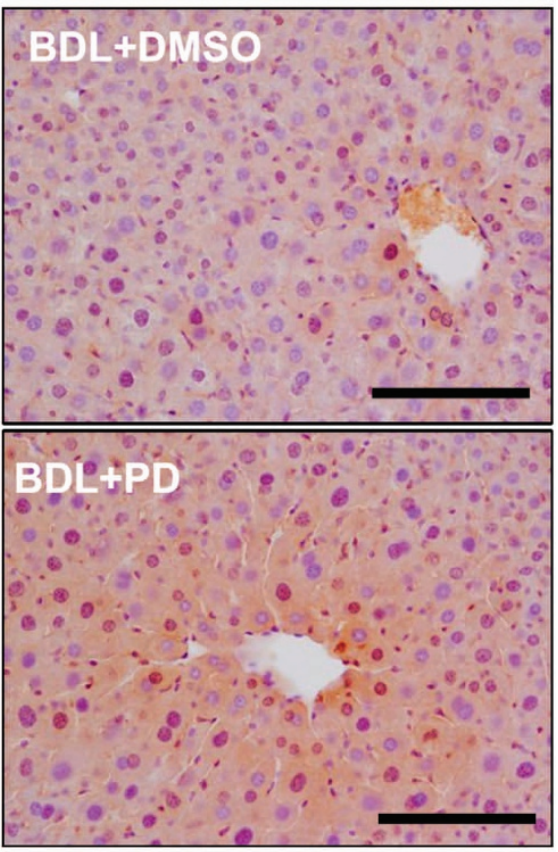

f
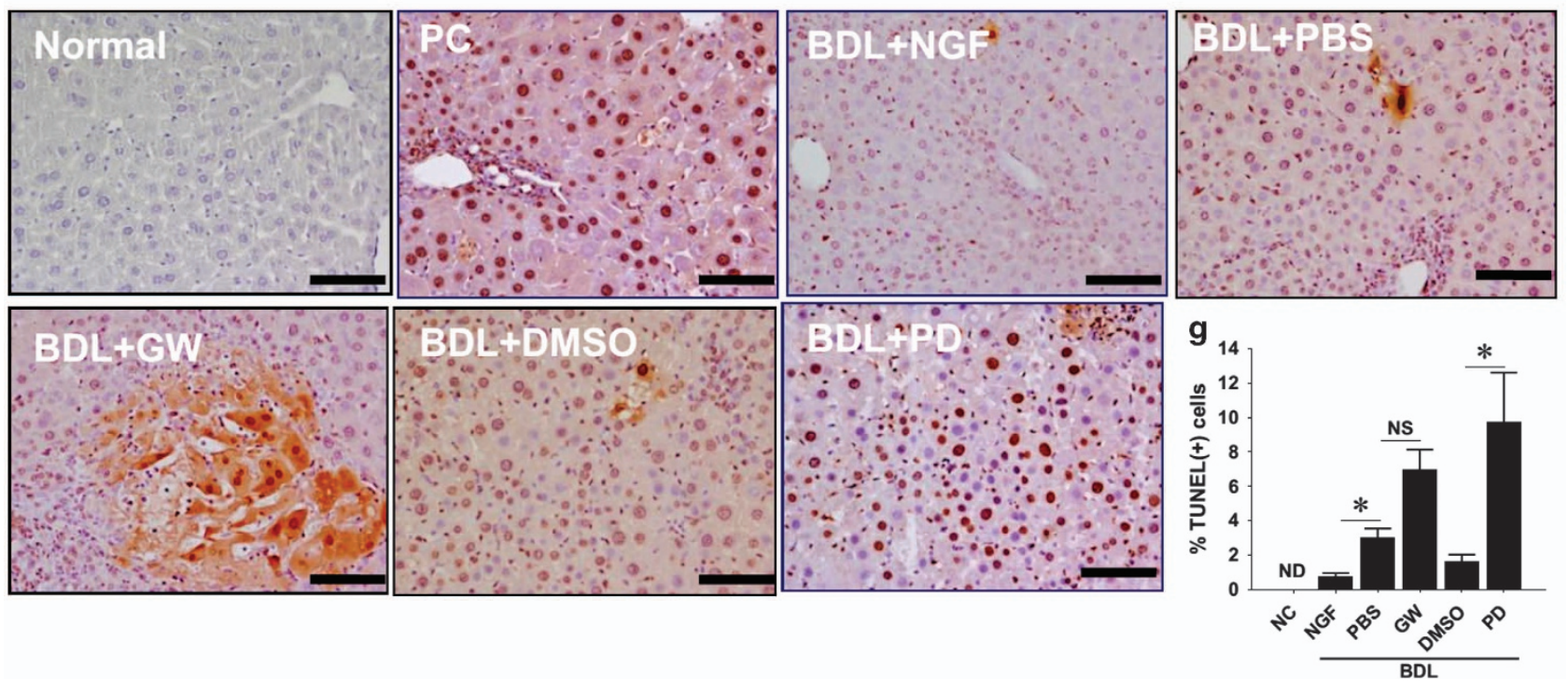
within intrahepatic biliary ducts are recognized hallmarks of hepatolithiasis, yet the mechanisms underlying cholestatic liver injury in hepatolithiasis remain unclear. Given that some hepatolithiasis patients still experience persistent liver damage after clearance of the stones, ${ }^{16}$ the pathophysiological mechanisms of hepatolithiasis might involve not only macroscopic impedance of bile flow by gallstones but also intrahepatic cholangiopathy, which is possibly induced by chronic inflammation. To this end, we believe the optimal therapy for hepatolithiasis should include strategies to alleviate long-term liver damage, in addition to the resumption of biliary flow. a

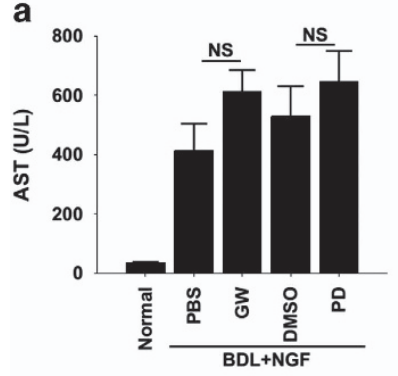

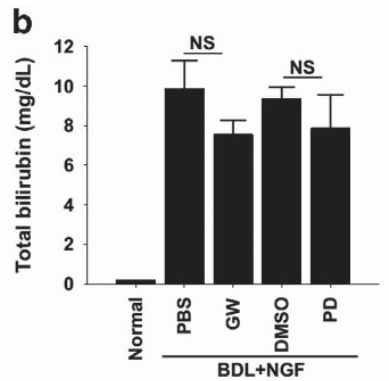

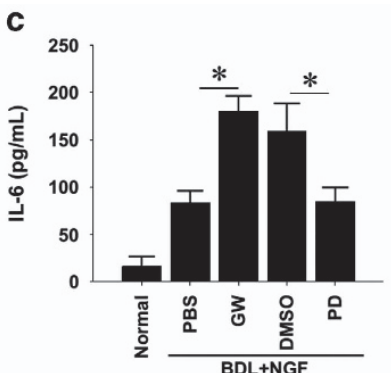

d

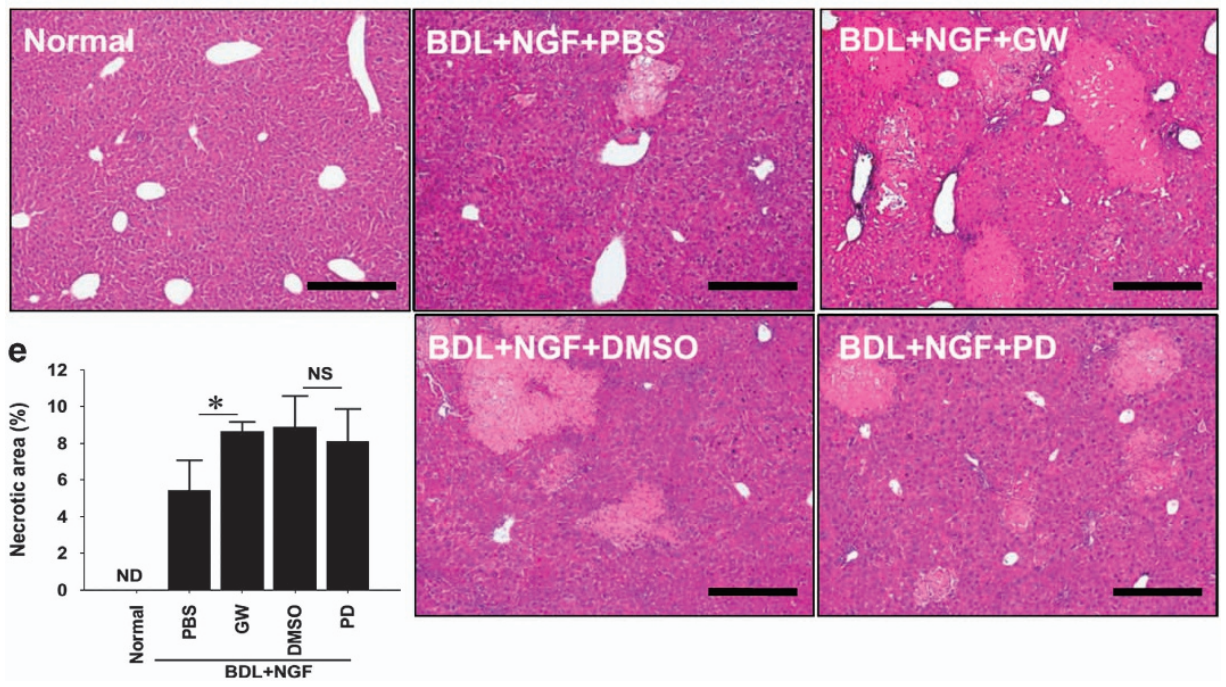

Figure 5 Effects of simultaneous administration with NGF and its receptor blockers on bile duct ligation (BDL)-induced mouse liver injury. The ICR mice receiving BDL surgery were intraperitoneally administered recombinant NGF $\left(1 \mathrm{mg} \mathrm{kg}^{-1} ; n=3\right)$ twice weekly and simultaneous daily injections of PBS ( $n=6)$, GW441756 (GW; $\left.1 \mathrm{mg} \mathrm{kg}^{-1} ; n=6\right)$, DMSO $(n=6)$ or PD90780 (PD; $\left.1 \mathrm{mg} \mathrm{kg}-1 ; n=6\right)$. At 14 days postoperation, serum levels of AST (a) and total bilirubin (b) were biochemically measured, whereas serum interleukin (IL)-6 levels (c) were analyzed by ELISA. (d) The representative H\&E staining results showing the necrotic regions in cholestasis-injured livers. Scale bar $=1000 \mu \mathrm{m}$. (e) Morphometric analysis of necrotic region areas. All data are shown as the mean \pm s.e.m. ${ }^{*} P<0.05$ between the indicated groups. ND, not detectable. NS, not significant.

Figure 4 Effects of TrkA and p75 NTR blocker treatments on bile duct ligation (BDL)-induced PARP cleavage and apoptosis of parenchymal hepatocytes. The mice receiving BDL surgery were intraperitoneally administered with either recombinant NGF $(n=6)$ twice weekly or PBS $(n=6)$, GW441756 (GW; $n=6)$, DMSO $(n=3)$ or PD90780 (PD; $n=6)$ daily. After 2 weeks of treatment, pooled liver extracts of each group were subjected to western blotting to detect both propeptides and cleaved forms of caspase-3 and PARP (a). Density analysis of the activation of caspase-3 (b) and PARP (c), shown as the ratios of cleavage to propeptide content, followed by normalization to negative control (NC) levels. IHC staining for active PARP peptides (d) and labeling index (e) quantitatively demonstrated that PD90780 administration potentiated the increase of PARP activation in BDL-treated mouse livers. (f) Representative microphotographs of TUNEL staining showing the apoptotic events in mouse liver sections. Scale bars $=50 \mu \mathrm{m}$. (g) Analysis of TUNELpositive parenchymal cells indicated that recombinant NGF administration exerted a protective effect, whereas treatment with a p75NTR antagonist, PD90780, increased apoptotic cell death in BDL-induced cholestatic injured livers. All data are shown as the mean \pm s.e.m. ${ }^{*} P<0.05$ between indicated groups. ND, not detectable. NS, not significant. 
a

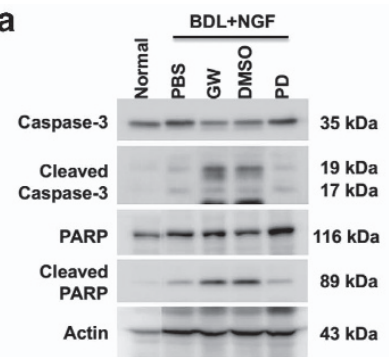

b

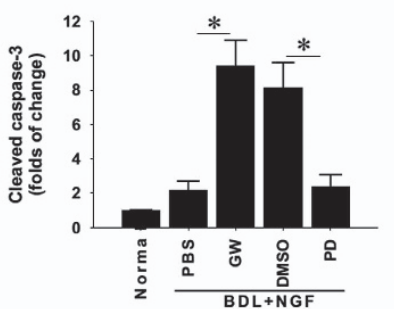

C

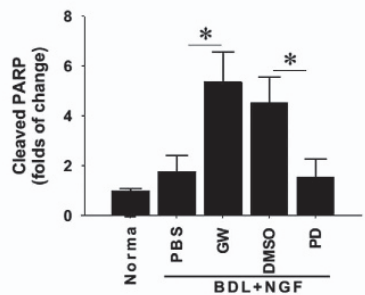

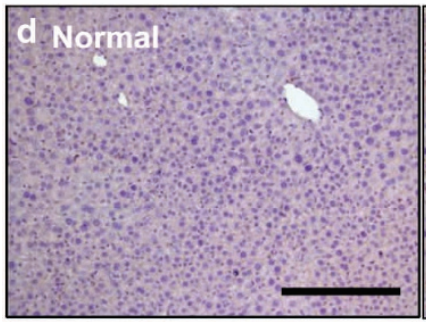

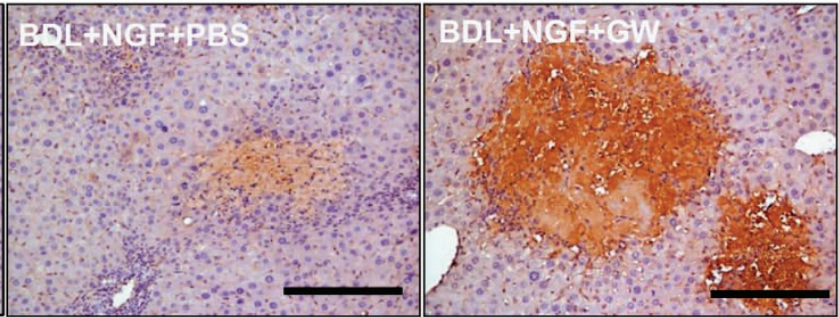

e
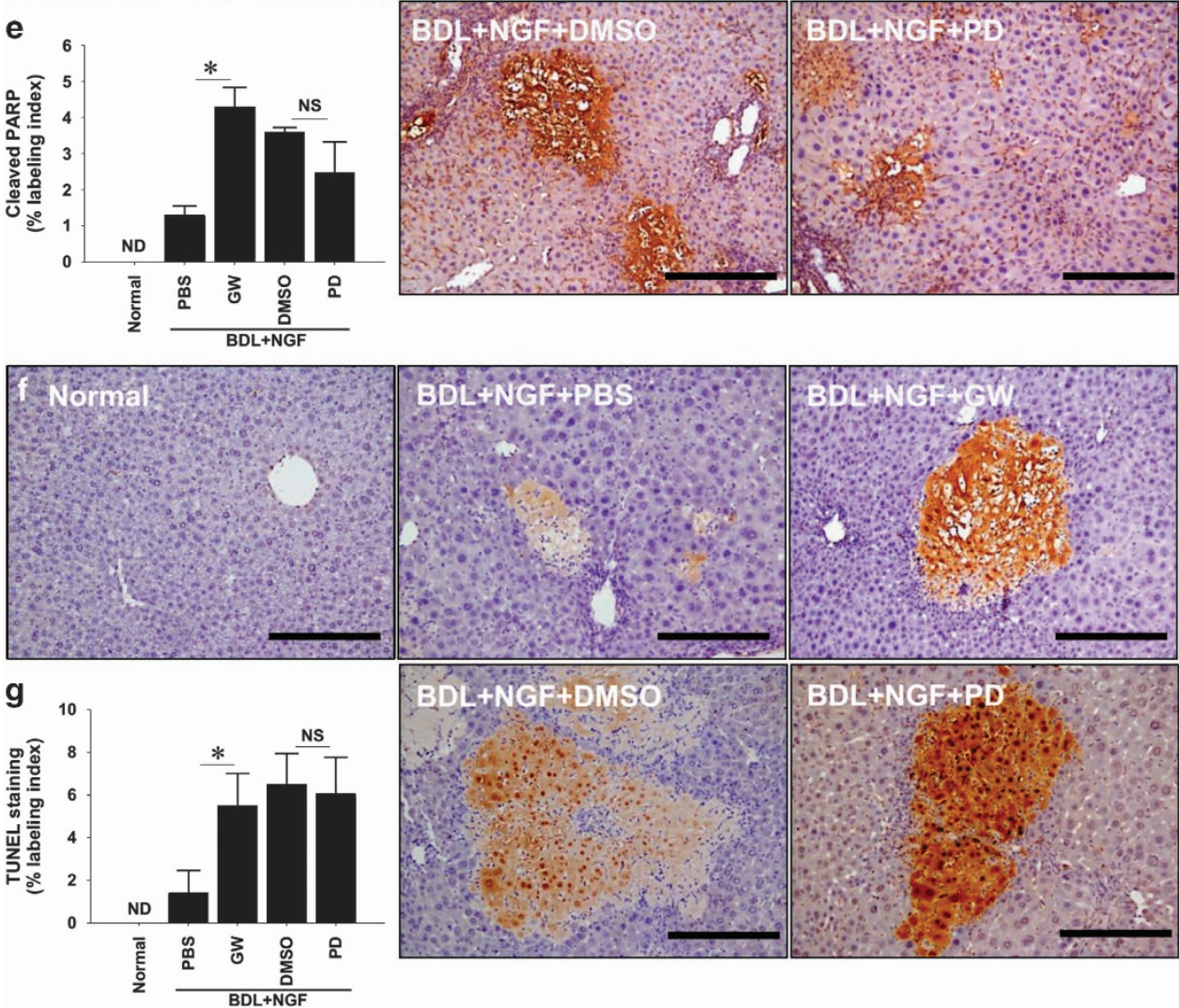

Figure 6 Effects of simultaneous administration with NGF and its receptor blockers on bile duct ligation (BDL)-induced apoptosis of parenchymal hepatocytes. The ICR mice receiving BDL surgery were intraperitoneally administered recombinant NGF (1 mg kg-1; $n=3)$ twice weekly and simultaneous daily injections of PBS $(n=6)$, GW441756 (GW; $\left.1 \mathrm{mg} \mathrm{kg}^{-1} ; n=6\right)$, DMSO ( $\left.n=6\right)$ or PD90780 (PD; $\left.1 \mathrm{mg} \mathrm{kg}^{-1} ; n=6\right)$. After 2 weeks of treatment, pooled liver extracts of each group were subjected to western blotting to detect both propeptides and cleaved forms of caspase-3 and PARP (a). The activation of caspase-3 (b) and PARP (c) was densitometrically analyzed and is shown as the cleavage-to-propeptide ratios and normalized to negative control (NC) levels. (d) Representative images of IHC staining for activated PARP in the mouse livers. (e) Quantitative measurement of PARP activation as shown by labeling index. (f) Representative microphotographs of TUNEL staining showing the apoptotic events in mouse liver sections. Scale bars $=50 \mu \mathrm{m}$. (g) Analysis of TUNEL-positive parenchymal cells indicated that GW treatment significantly increased apoptotic cell death in BDL-induced cholestatic injured livers. All data are shown as the mean \pm s.e.m. ${ }^{*} P<0.05$ between indicated groups. ND, not detectable. NS, not significant. 

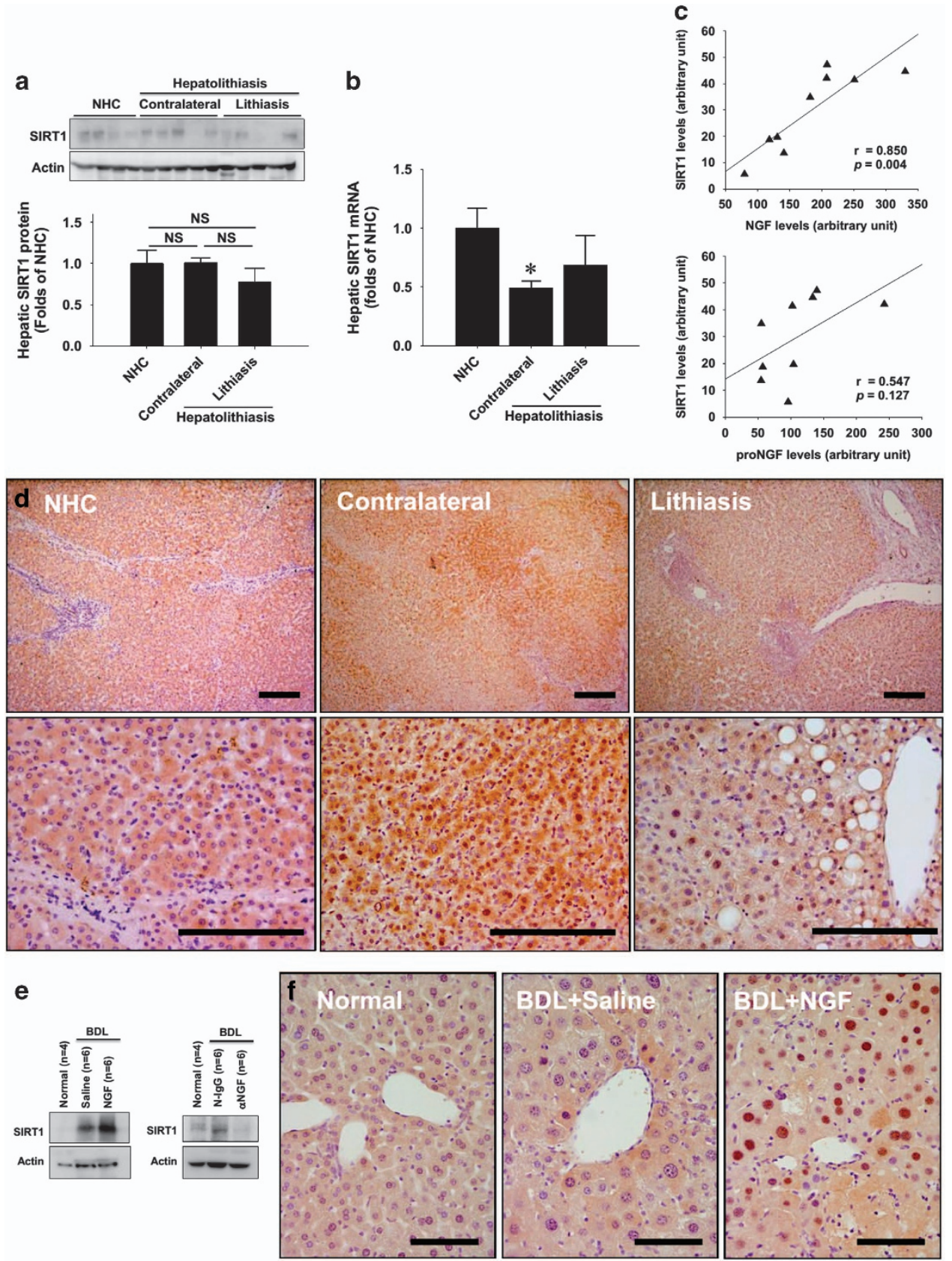

Figure 7 Expression of SIRT1 in human hepatolithiasis and mouse cholestatic livers. (a) Western blotting detection of hepatic SIRT1 expression in human non-hepatolithiasis controls (NHC, $n=4$ ), as well as lithiasis and contralateral regions in human hepatolithiasis livers $(n=5)$. NS, not significant. (b) RT-qPCR detection of SIRT1 mRNA in human hepatolithiasis livers. All data are shown as the mean \pm s.e.m. ${ }^{*} P<0.05$ compared with NHC. (c) Spearman's correlation analysis of all of the human liver specimens suggested a positive correlation between hepatic SIRT1 and NGF/proNGF expression. (d) SIRT1 IHC staining showing both nuclear and cytoplasmic localization of SIRT1 peptides in hepatocytes of human NHC and hepatolithiasis livers. Bar $=200 \mu \mathrm{m}$. (e) Upregulation of SIRT1 by NGF treatment and its abolishment by NGF-neutralizing antibodies in a mouse model of bile duct ligation (BDL) surgery-induced cholestatic liver injury. Mouse livers were collected 14 days post-BDL surgery. (f) SIRT1 IHC staining of mouse livers that received BDL surgery followed by NGF or saline administration for 14 days. Bar $=100 \mu \mathrm{m}$.

NGF and its precursor, proNGF, belong to the family of neurotrophins, which are essential for neuron survival, differentiation and cell death. It has been well documented that hepatocytes could synthesize NGF in various types of liver injury, such as fibrosis, ${ }^{17-19}$ chemical injury ${ }^{18}$ and BDL. ${ }^{8,20}$
Both NGF and proNGF could bind to the two NGFRs, TrkA and p75NTR. Previous studies have also demonstrated the expression of NGFRs in cholangiocytes ${ }^{20}$ hepatic stellate cells, ${ }^{21-23}$ other non-parenchymal cells ${ }^{24,25}$ and hepatocytes. ${ }^{15}$ Several regulatory roles of NGF and proNGF have been 
a
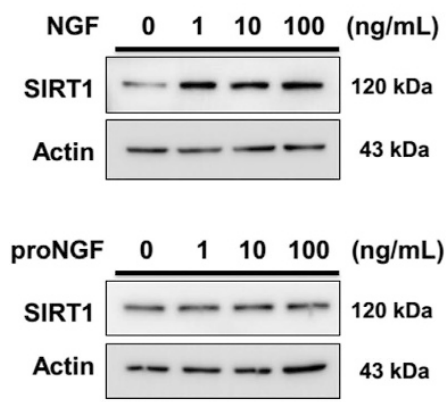

b

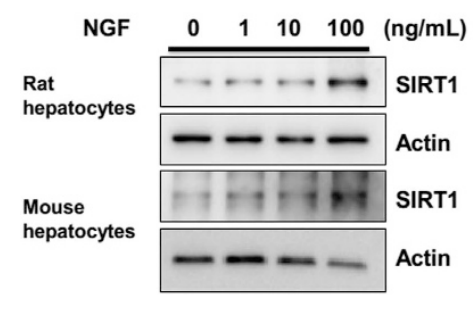

C

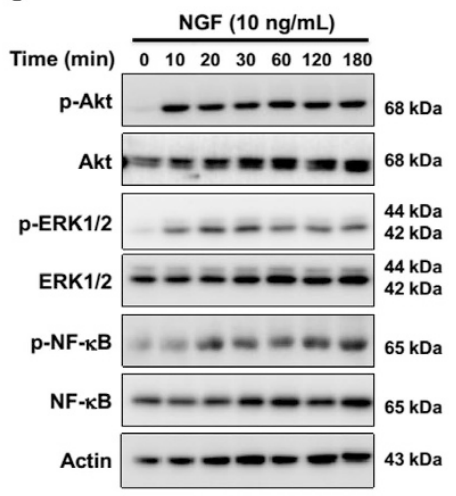

d

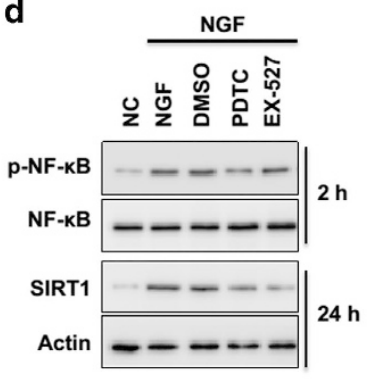

e
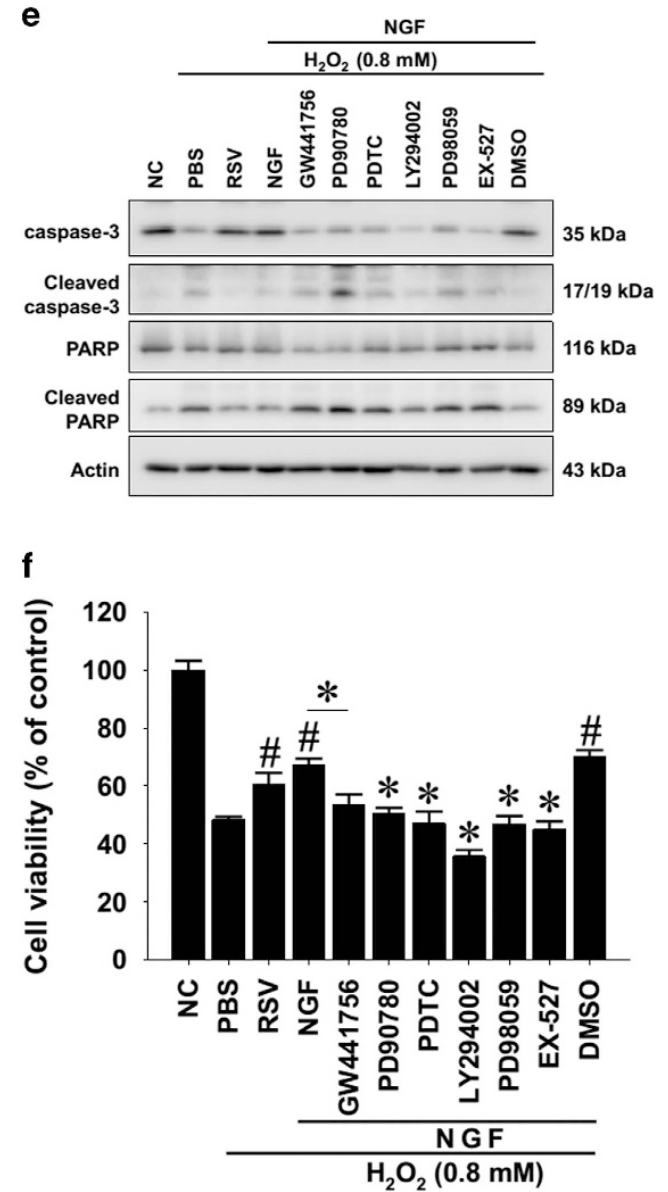

Figure 8 Involvement of NGF-induced SIRT1 upregulation in hepatocytes under oxidative stress. (a) Huh-7 hepatocytes were treated with either NGF or proNGF for $24 \mathrm{~h}$. Western blotting detection indicated that NGF, but not proNGF, upregulated SIRT1 expression in human hepatocytes. (b) SIRT1 upregulation by NGF stimulation in cultured rat and mouse primary hepatocytes. (c) Kinetic profiles of NGF-mediated signaling activation. Huh-7 cells were treated with NGF for the indicated durations and measured by western blotting. (d) Involvement of NF-KB and SIRT1 activities in the NGF-induced SIRT1 upregulation. Huh-7 cells were pretreated with $10 \mu \mathrm{M}$ PDTC or $20 \mu \mathrm{M}$ EX-527 for $2 \mathrm{~h}$, followed by 2 or $24 \mathrm{~h}$ of NGF stimulation. (e) Effects of NGFR blockers, kinase inhibitors and SIRT1 modulators on oxidative stress-activated pro-apoptotic machinery. Huh-7 cells were pretreated either with $20 \mu \mathrm{m}$ resveratrol (RSV; a SIRT1 agonist) or with $10 \mathrm{ng} \mathrm{ml}^{-1} \mathrm{NGF}$ for $24 \mathrm{~h}$ in the presence of $10 \mu \mathrm{m}$ NGFR blockers (GW441756 and PD90780), $10 \mu \mathrm{m}$ kinase blockers (PDTC, LY294002

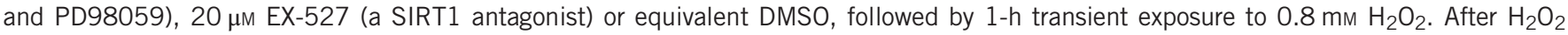
removal and another 4-h incubation, protein lysates were collected for western blotting detection of caspase-3 and PARP. The density analysis data are shown in Supplementary Figure S6. (f) Effects of NGFR blockers, kinase inhibitors and SIRT1 modulators on oxidative stress-induced hepatotoxicity as measured by MTT-based cell viability assay. All data are shown as the mean \pm s.d. of three independent experiments. ${ }^{*} P<0.05$ vs DMSO solvent control or between the indicated groups; ${ }^{\#} P<0.05$ vs the $\mathrm{H}_{2} \mathrm{O}_{2}$ treatment group.

proposed in these types of cells, including proliferation of intrahepatic biliary epithelium, ${ }^{20}$ apoptosis of hepatic activated stellate cells, ${ }^{23}$ angiogenesis ${ }^{25}$ and lipid metabolism of hepatocytes. ${ }^{15}$ In the present study, we only detected upregulation of p75NTR, but not of NGF or proNGF, in livers with hepatolithiasis. As NGF and proNGF are soluble molecules, upregulation of NGF and/or proNGF signaling does not necessarily indicate that more molecules exist within the liver tissues. Moreover, soluble NGF/proNGF can be delivered into the liver via the circulation and subsequently exert their physiological effects. Indeed, our previous study showed increased serum NGF levels in animals undergoing BDL surgery. ${ }^{8}$
ProNGF is posttranslationally processed to form mature $\mathrm{NGF}^{26}$ It is believed that, in the central nervous system, the proNGF molecule predominates, whereas little or no mature NGF is present. ${ }^{27}$ It seems that both NGF and proNGF have dual abilities to promote survival and cell death, depending on the cell type and the expression of different receptors. ${ }^{27,28}$ Our present work focused on delineating the role of the NGF signaling pathway in cholestatic liver injury but did not completely exclude the pathophysiological significance of proNGF. Although our findings demonstrated that proNGF did not upregulate SIRT1 expression in hepatocytes, it exerted hepatoprotective effects, similar to NGF, in vitro. Furthermore, both TrkA and p75NTR signaling could be involved in the 
pro-survival effects of exogenous proNGF treatment. It would therefore be interesting to conduct a future study to clarify the role of proNGF in livers.

It remains controversial whether hepatocytes express NGFRs and therefore could be regulated by NGF in an autocrine manner. In animal models, TrkA expression has been found in hepatocytes undergoing acute oxidative injury. ${ }^{29}$ Hepatocytes in human cirrhotic livers also expressed TrkA. ${ }^{30}$ However, it was argued that hepatocytes only expressed NGF, but not TrkA, in healthy livers or during profibrotic liver injury. ${ }^{18,30}$ P75NTR has been reported to be expressed in hepatocytes. ${ }^{15,31}$ Moreover, one recent study demonstrated that hepatocytes expressed both NGF and p75NTR in human chronic liver injury samples. ${ }^{31}$ In agreement with previous studies, we found that hepatocytes expressed both TrkA and p75NTR, at least in diseased livers with cholestasis or viral hepatitis (non-cholestatic livers), supporting an autocrine role of NGF signaling via these two receptors in hepatocytes.

It has traditionally been believed that binding of NGF to TrkA leads to cell survival, whereas binding of proNGF to p75NTR promotes cell death in neural cells. ${ }^{28,32}$ However, more studies have suggested that both NGF (via p75NTR) and proNGF (via TrkA) could also promote cell survival, depending on the cell type and physiological stage. ${ }^{27,33,34}$ In the present study, the in vitro results suggested that both $\operatorname{TrkA}$ and p75NTR were involved in hepatocyte survival under oxidative stress. Moreover, in the absence of exogenous NGF/proNGF, blockage of p75NTR deteriorated hepatocyte injury in vitro and in vivo, suggesting that p75NTR signaling was essential for alleviating liver injury in cholestasis.

Although TrkA blocker alone did not aggravate liver injury in BDL animals, it should be noted that concomitant administration of TrkA blocker, but not p75NTR blocker, counteracted the hepatoprotective effect of NGF in vivo, perhaps in part because of the existence of multiple ligands (for example, NGF and proNGF) of TrkA and p75NTR in vivo. As NGF has a high affinity for TrkA and a low affinity for p75NTR, the significance of TrkA signaling might only be revealed when NGF is sufficiently abundant. Indeed, how these two receptors interact to determine cell survival is difficult to decipher, largely because both can signal independently or coincidentally, depending on the cell type, number of different ligands and physiological conditions. ${ }^{35}$ Taken together, our results suggested that the TrkA pathway might be critical for the therapeutic effect of additional NGF treatment in BDL animals.

Both TrkA and p75NTR signaling could activate a number of pro-survival signaling machineries. The pro-survival pathways downstream of TrkA include the signaling mediated by phosphoinositide-3 kinase/Akt, ${ }^{36} \mathrm{ERK}^{37}$ and NF-кB. ${ }^{38}$ By contrast, p75NTR signaling could also promote cell survival via the protein kinase $\mathrm{C} \zeta{ }^{39}$ NF- $\kappa B^{36,37,39}$ and phosphoinositide-3 kinase pathways. ${ }^{38}$ The present study demonstrated that NGF induced NF- $\kappa \mathrm{B}$ pathways in Huh-7 hepatocytes, and NF- $\kappa \mathrm{B}$ activity had an important role in the regulation of SIRT1 expression therein, whereas pharmacological blockade of NF- $\kappa B$ counteracted the in vitro hepatoprotective effects of NGF and
proNGF. It will therefore be interesting to further evaluate the crosstalk among TrkA, p75NTR, NF- $\kappa$ B and phosphoinositide-3 kinase in hepatocytes.

SIRT1 belongs to the family of NAD+-dependent class III histone deacetylases and is able to deacetylate several nuclear proteins, such as histones, transcription factors and cofactors. ${ }^{40}$ However, cytoplasmic SIRT1 could also directly interact with cytoplasmic proteins ${ }^{41}$ and therefore modulate important signaling pathways, including NGF signaling. ${ }^{42}$ SIRT1 has been reported to be involved in cell differentiation, survival and metabolism. ${ }^{43,44}$ In liver tissues, downregulation of SIRT1 contributed to D-galactosamine/lipopolysaccharide-induced liver injury, whereas resveratrol (a SIRT1 agonist) prevented this liver injury. ${ }^{45}$ SIRT1 was also proposed to be a potential therapeutic target in nonalcoholic fatty liver disease. ${ }^{46}$ The present study proposed that SIRT1 has a role in cholestatic liver injury, possibly by modulating NGF signaling.

There were some limitations, however, of the present study. First, Huh-7 cells are usually regarded as a cell line of human hepatocellular carcinoma, instead of normal hepatocytes. The in vitro mechanistic findings of this study partially supported our claims, which could be reproduced in normal human hepatocytes. Second, the BDL animal model does not completely represent the cholestatic liver injury of hepatolithiasis, which might last for months or even years. Last, we did not study all of the ligands of p75NTR and TrkA and we only focused on NGF signaling in the present study. It should be noted that ligands other than NGF and proNGF might have physiological roles in cholestatic liver injury. The pathophysiological significance of these ligands merits further investigation.

In conclusion, we reported that NGF, proNGF and their receptors were expressed in the hepatocytes of human hepatolithiasis liver samples. Moreover, exogenous NGF administration could inhibit hepatocyte apoptosis through the activation of both TrkA and p75NTR in cholestatic animal models. The hepatoprotective effects of NGF were closely associated with the upregulation of the antiaging transcription factor, SIRT1. The NGF/SIRT1 axis could be regarded as a potential target for the treatment of cholestatic disease.

\section{CONFLICT OF INTEREST}

The authors declare no conflict of interest.

\section{ACKNOWLEDGEMENTS}

We thank Yi-Chun Chen, Ya-Chun Lee and Tzu-Shan Chen for their technical support and statistical assistance from the Bio-Bank and Data Bank Centers in the Department of Medical Research, E-Da Hospital. This study was supported by the Ministry of Science and Technology, Taiwan (Grant no. MOST105-2314-B-650-007) and the E-Da Hospital, Taiwan (Grant nos. EDAHP-102001, -103013, -104007, -105004 and EDPJ-105065).

Author contributions: M-ST, P-HL, C-KS, T-CC, Y-CL, I-WC, P-HC and Y-HK conceived of the experiments. I-WC analyzed the human specimens. C-KS, Y-CL and P-HC performed the animal experiments. $\mathrm{T}-\mathrm{CC}$ and $\mathrm{P}-\mathrm{HC}$ performed the in vitro experiments and statistical analyses. All the authors were involved in writing the paper and had final approval of the submitted and published versions. 


\section{PUBLISHER'S NOTE}

Springer Nature remains neutral with regard to jurisdictional claims in published maps and institutional affiliations.

1 Vetrone G, Ercolani G, Grazi GL, Ramacciato G, Ravaioli M, Cescon M et al. Surgical therapy for hepatolithiasis: a Western experience. J Am Coll Surg 2006; 202: 306-312.

2 Tabrizian P, Jibara G, Shrager B, Schwartz ME, Roayaie S. Hepatic resection for primary hepatolithiasis: a single-center Western experience. J Am Coll Surg 2012; 215: 622-626.

3 Chen DW, Tung-Ping Poon R, Liu CL, Fan ST, Wong J. Immediate and longterm outcomes of hepatectomy for hepatolithiasis. Surgery 2004; 135: 386-393.

4 Tazuma S. Gallstone disease: epidemiology, pathogenesis, and classification of biliary stones (common bile duct and intrahepatic). Best Pract Res Clin Gastroenterol 2006; 20: 1075-1083.

5 Nakayama F, Soloway RD, Nakama T, Miyazaki K, Ichimiya H, Sheen PC et al. Hepatolithiasis in East Asia. Retrospective study. Dig Dis Sci 1986; 31: 21-26.

6 Mori T, Sugiyama M, Atomi Y. Gallstone disease: management of intrahepatic stones. Best Pract Res Clin Gastroenterol 2006; 20: 1117-1137.

7 Yang T, Lau WY, Lai EC, Yang LQ, Zhang J, Yang GS et al. Hepatectomy for bilateral primary hepatolithiasis: a cohort study. Ann Surg 2010; 251: 84-90.

8 Tsai MS, Lin YC, Sun CK, Huang SC, Lee PH, Kao YH. Up-regulation of nerve growth factor in cholestatic livers and its hepatoprotective role against oxidative stress. PLOS ONE 2014; 9: e112113.

9 Ljubuncic P, Tanne Z, Bomzon A. Evidence of a systemic phenomenon for oxidative stress in cholestatic liver disease. Gut 2000; 47: 710-716.

10 Tsai MS, Su YH, Ho MC, Liang JT, Chen TP, Lai HS et al. Clinicopathological features and prognosis in resectable synchronous and metachronous colorectal liver metastasis. Ann Surg Oncol 2007; 14: 786-794.

$11 \mathrm{Kao}$ YH, Chen CL, Jawan B, Chung YH, Sun CK, Kuo SM et al. Upregulation of hepatoma-derived growth factor is involved in murine hepatic fibrogenesis. J Hepatol 2010; 52: 96-105.

12 Pinkse GG, Voorhoeve MP, Noteborn M, Terpstra OT, Bruijn JA, De Heer E. Hepatocyte survival depends on betal-integrin-mediated attachment of hepatocytes to hepatic extracellular matrix. Liver Int 2004; 24: 218-226.

13 Chiba T, Yokosuka O, Fukai K, Kojima H, Tada M, Arai M et al. Cell growth inhibition and gene expression induced by the histone deacetylase inhibitor, trichostatin A, on human hepatoma cells. Oncology 2004; 66: 481-491.

14 Khan RS, Fonseca-Kelly Z, Callinan C, Zuo L, Sachdeva MM, Shindler KS. SIRT1 activating compounds reduce oxidative stress and prevent cell death in neuronal cells. Front Cell Neurosci 2012; 6: 63.

15 Pham DD, Do HT, Bruelle C, Kukkonen JP, Eriksson O, Mogollon I et al. p75 neurotrophin receptor signaling activates sterol regulatory elementbinding protein-2 in hepatocyte cells via p38 mitogen-activated protein kinase and caspase-3. J Biol Chem 2016; 291: 10747-10758.

16 Chijiiwa K, Kameoka N, Komura M, Yamasaki T, Noshiro H, Nakano K. Hepatic resection for hepatolithiasis and long-term results. J Am Coll Surg 1995; 180: 43-48

17 Trim N, Morgan S, Evans M, Issa R, Fine D, Afford S et al. Hepatic stellate cells express the low affinity nerve growth factor receptor p 75 and undergo apoptosis in response to nerve growth factor stimulation. Am J Pathol 2000; 156: 1235-1243.

18 Oakley F, Trim N, Constandinou CM, Ye W, Gray AM, Frantz G et al. Hepatocytes express nerve growth factor during liver injury: evidence for paracrine regulation of hepatic stellate cell apoptosis. Am J Pathol 2003; 163: 1849-1858.

19 Kendall TJ, Hennedige S, Aucott RL, Hartland SN, Vernon MA, Benyon RC et al. p75 Neurotrophin receptor signaling regulates hepatic myofibroblast proliferation and apoptosis in recovery from rodent liver fibrosis. Hepatology 2009; 49: 901-910.

20 Gigliozzi A, Alpini G, Baroni GS, Marucci L, Metalli VD, Glaser SS et al. Nerve growth factor modulates the proliferative capacity of the intrahepatic biliary epithelium in experimental cholestasis. Gastroenterology 2004; 127: $1198-1209$.
21 Passino MA, Adams RA, Sikorski SL, Akassoglou K. Regulation of hepatic stellate cell differentiation by the neurotrophin receptor p75NTR. Science 2007; 315: 1853-1856.

22 Cassiman D, Denef C, Desmet VJ, Roskams T. Human and rat hepatic stellate cells express neurotrophins and neurotrophin receptors. Hepatology 2001; 33: 148-158.

23 Asai K, Tamakawa S, Yamamoto M, Yoshie M, Tokusashi Y, Yaginuma Y et al. Activated hepatic stellate cells overexpress p75NTR after partial hepatectomy and undergo apoptosis on nerve growth factor stimulation. Liver Int 2006; 26: 595-603.

24 Suzuki K, Tanaka M, Watanabe N, Saito S, Nonaka H, Miyajima A. p75 Neurotrophin receptor is a marker for precursors of stellate cells and portal fibroblasts in mouse fetal liver. Gastroenterology 2008; 135: 270-281. e3.

25 Kishibe K, Yamada Y, Ogawa K. Production of nerve growth factor by mouse hepatocellular carcinoma cells and expression of TrkA in tumor-associated arteries in mice. Gastroenterology 2002; 122: 1978-1986.

26 Seidah NG, Benjannet S, Pareek S, Savaria D, Hamelin J, Goulet B et al. Cellular processing of the nerve growth factor precursor by the mammalian pro-protein convertases. Biochem J 1996; 314(Pt 3): 951-960.

27 Fahnestock M, Yu G, Michalski B, Mathew S, Colquhoun A, Ross GM et al. The nerve growth factor precursor proNGF exhibits neurotrophic activity but is less active than mature nerve growth factor. J Neurochem 2004; 89: 581-592.

28 Lee R, Kermani P, Teng KK, Hempstead BL. Regulation of cell survival by secreted proneurotrophins. Science 2001; 294: 1945-1948.

29 Valdovinos-Flores C, Gonsebatt ME. Nerve growth factor exhibits an antioxidant and an autocrine activity in mouse liver that is modulated by buthionine sulfoximine, arsenic, and acetaminophen. Free Radic Res 2013; 47: 404-412.

30 Rasi G, Serafino A, Bellis L, Lonardo MT, Andreola F, Zonfrillo M et al. Nerve growth factor involvement in liver cirrhosis and hepatocellular carcinoma. World J Gastroenterol 2007; 13: 4986-4995.

31 Amoras Eda S, Gomes ST, Freitas FB, Santana BB, Ishak G, de Araujo MT et al. NGF and P75NTR gene expression is associated with the hepatic fibrosis stage due to viral and non-viral causes. PLOS ONE 2015; 10 e0121754.

32 Barrett GL. The p75 neurotrophin receptor and neuronal apoptosis. Prog Neurobiol 2000; 61: 205-229.

33 DeFreitas MF, McQuillen PS, Shatz CJ. A novel p75NTR signaling pathway promotes survival, not death, of immunopurified neocortical subplate neurons. J Neurosci 2001; 21: 5121-5129.

34 Mamidipudi V, Wooten MW. Dual role for p75(NTR) signaling in survival and cell death: can intracellular mediators provide an explanation? J Neurosci Res 2002; 68: 373-384.

35 Culmsee C, Gerling N, Lehmann M, Nikolova-Karakashian M, Prehn JH, Mattson MP et al. Nerve growth factor survival signaling in cultured hippocampal neurons is mediated through TrkA and requires the common neurotrophin receptor P75. Neuroscience 2002; 115: $1089-1108$.

36 Demarchi F, Bertoli C, Greer PA, Schneider C. Ceramide triggers an NF-kappaB-dependent survival pathway through calpain. Cell Death Differ 2005; 12: 512-522.

37 Hamanoue M, Middleton G, Wyatt S, Jaffray E, Hay RT, Davies AM. p75-mediated NF-kappaB activation enhances the survival response of developing sensory neurons to nerve growth factor. Mol Cell Neurosci 1999; 14: $28-40$.

38 Gomez-Munoz A, Kong JY, Parhar K, Wang SW, Gangoiti P, Gonzalez M et al. Ceramide-1-phosphate promotes cell survival through activation of the phosphatidylinositol 3-kinase/protein kinase B pathway. FEBS Lett 2005; 579: 3744-3750.

39 Wang YM, Seibenhener ML, Vandenplas ML, Wooten MW. Atypical PKC zeta is activated by ceramide, resulting in coactivation of NF-kappaB/JNK kinase and cell survival. J Neurosci Res 1999; 55: 293-302.

40 Imai S, Armstrong CM, Kaeberlein M, Guarente L. Transcriptional silencing and longevity protein Sir2 is an NAD-dependent histone deacetylase. Nature 2000; 403: 795-800.

41 Law IK, Liu L, Xu A, Lam KS, Vanhoutte PM, Che CM et al. Identification and characterization of proteins interacting with SIRT1 and SIRT3: implications in the anti-aging and metabolic effects of sirtuins. Proteomics 2009; 9: 2444-2456.

42 Sugino T, Maruyama M, Tanno M, Kuno A, Houkin K, Horio $Y$. Protein deacetylase SIRT1 in the cytoplasm promotes nerve growth 
factor-induced neurite outgrowth in PC12 cells. FEBS Lett 2010; 584: 2821-2826.

43 Haigis MC, Guarente LP. Mammalian sirtuins-emerging roles in physiology, aging, and calorie restriction. Genes Dev 2006; 20: 2913-2921.

44 Longo VD, Kennedy BK. Sirtuins in aging and age-related disease. Cell 2006; 126: 257-268.

45 Kemelo MK, Wojnarova L, Kutinova Canova N, Farghali H. D-galactosamine/lipopolysaccharide-induced hepatotoxicity downregulates sirtuin 1 in rat liver: role of sirtuin 1 modulation in hepatoprotection. Physiol Res 2014; 63: 615-623.

46 Colak Y, Ozturk O, Senates E, Tuncer I, Yorulmaz E, Adali G et al. SIRT1 as a potential therapeutic target for treatment of nonalcoholic fatty liver disease. Med Sci Monit 2011; 17: HY5-HY9. (c) (i) (s) $€$ This work is licensed under a Creative Commons Attribution-NonCommercial-NoDerivs 4.0 International License. The images or other third party material in this article are included in the article's Creative Commons license, unless indicated otherwise in the credit line; if the material is not included under the Creative Commons license, users will need to obtain permission from the license holder to reproduce the material. To view a copy of this license, visit http://creativecommons.org/licenses/by-nc-nd/4.0/

(C) The Author(s) 2018

Supplementary Information accompanies the paper on Experimental \& Molecular Medicine website (http://www.nature.com/emm) 